Table 2. Peirce's measure of predictive success for comparison of the three CV measures of colleges from the three leading states in the NIRF top 100

\begin{tabular}{lccc}
\hline Peirce's measure & NIRF - perception & $X$ - perception & $X-$ NIRF \\
\hline Tamil Nadu 35 & 0.50 & 0.38 & 0.37 \\
Delhi 29 & 0.65 & 0.36 & 0.52 \\
Kerala 18 & 0.00 & 0.00 & 0.20 \\
\hline
\end{tabular}

positive and false negative quadrants is taken into account. In all three states, and overall for the whole country, the perception score considerably underestimates most institutions and the NIRF score generally overestimates them.

We examined the 2019 scores from NIRF for the top 100 colleges in India from the $\mathrm{CV}$ point of view. The NIRF exercise provides a final score (the NIRF score) from five broad parameters for participating institutions. One parameter is an observed variable and is a peerreview-based perception score. Using the TLR parameter as a proxy for teaching and learning resources input, and the RPC and GO parameters as proxies for teaching and research outputs or outcomes, we independently computed a second-order $X$-score. The NIRF scores and the $X$-scores are latent variables that emerge from mathematical models. The three scores are compared in the context of $\mathrm{CV}$, and weaknesses and biases can be recognized while validating such multi-dimensional evaluation exercises. Peirce's quantitative measure of predictive success is used to determine if one construct measure can predict the other.

One interesting but unsurprising takeaway is that the perception scores favour colleges based in Delhi and that this bias is carried over to the NIRF scores as well. Again, not unsurprisingly, the perception scores underestimate the performance of most of the institutions which are at the lower end of the spectrum. The bias is particularly felt by the colleges from Kerala.

Precisely the reverse picture emerges from the NIRF scores. Now, the performance of institutions at the lower end of the spectrum is shored up considerably-these scores are noticeably higher than those computed from the $X$-scores model. The $X$-score which is based on an input-output model, where the input term, TLR appears in the denominator, may give a better representation of reality than the NIRF model, where TLR is added to the output parameters to arrive at a final score.

1. All India survey on higher education (2016-17); http://aishe. nic.in/aishe/viewDocument.action?documentId=239 (accessed on 12 April 2019).

2. Prathap, G., Danger of a single score: NIRF rankings of colleges. Curr. Sci., 2017, 113(4), 550-553.

3. Savithri, S. and Prathap, G., Indian and Chinese higher education institutions compared using an end-to-end evaluation. Curr. Sci., 2015, 108(10), 1922-1926.
4. Peirce, C. S., The numerical measure of the success of predictions. Science, 1884, 4(93), 453-454.

5. Bornmann, L., Tekles, A. and Leydesdorff, L., How well does I3 perform for impact measurement compared to other bibliometric indicators? The convergent validity of several (field-normalized) indicators. Scientometrics, 2019; https://doi.org/10.1007/s11192019-03071-6.

6. Prathap, G., Making scientometric and econometric sense out of NIRF 2017 data. Curr. Sci., 2017, 113(7), 1420-1423.

7. Prathap, G., Totalized input-output assessment of research productivity of nations using multi-dimensional input and output. Scientometrics, 2018, 115(1), 577-583.

8. Prathap, G., The Energy-Exergy-Entropy (or EEE) sequences in bibliometric assessment. Scientometrics, 2011, 87(3), 515-524.

9. Cronbach, L. J. and Meehl, P. E., Construct validity in psychological tests. Psychol. Bull., 1955, 52(4), 281-302; doi:10.1037/h0040957.

10. Cook, T. D. and Campbell, D. T., Quasi-Experimentation: Design \& Analysis Issues in Field Settings, Houghton Mifflin, Boston, USA, 1979.

11. Bornmann, L. and Daniel, H. D., Convergent validation of peer review decisions using the $\mathrm{h}$ index: extent of and reasons for type I and type II errors. J. Informetr., 2007, 1, 204-213.

12. Seglen, P. O., Why the impact factor of journals should not be used for evaluating research. Br. Med. J., 1997, 314, 498-502.

13. Smith, S. D., Is an article in a top journal a top article? Financ. Manage., 2004, 133-149.

14. Prathap, G., Mini, S. and Nishy, P., Does high impact factor successfully predict future citations? An analysis using Peirce's measure. Scientometrics, 2016, 108(3), 1043-1047. doi: $10.18520 / \mathrm{cs} / \mathrm{v} 117 / \mathrm{i} 6 / 1079-1083$

\section{Exploring the modulatory effect of albumin on calcium phosphate crystallization}

\author{
Priyadarshini ${ }^{1, *}$, Devesh Raizada ${ }^{1}$, \\ Pragya Kumar ${ }^{1}$, Tanya Singh ${ }^{1}$, Trisha Pruthi ${ }^{1}$, \\ Abhishek Negi ${ }^{1}$, Lokesh Nigam ${ }^{2}$ and \\ Naidu Subbarao ${ }^{2}$ \\ ${ }^{1}$ Department of Biotechnology, Jaypee Institute of Information \\ Technology, Noida 201 307, India \\ ${ }^{2}$ School of Computational and Integrative Sciences, \\ Jawaharlal Nehru University, New Delhi 110 067, India
}

Urolithiasis, also known as renal lithiasis, is a commonly occurring disease in humans. Calculi are formed in the urinary tract, including the tubular region of the kidney. Calcium phosphate crystals, especially hydroxyapatite, are responsible for nidus formation in the more commonly occurring calcium oxalate kidney stones. In the present study, effect of different concentrations of human serum albumin on

*For correspondence. (e-mail: priyadarshini@jiit.ac.in) 
nucleation, growth and demineralization of calcium phosphate crystallization using growth assay system and its confirmation with in silico study have been analysed. Human serum albumin shows inhibitory effect on nucleation, while it shows both stimulatory as well as inhibitory activity on the growth of calcium phosphate crystals. The results suggest that albumin plays a role in both inhibition as well as promotion of calcium phosphate crystallization. The in silico study indicates involvement of Tyr-161, Arg-222 and Lys195 and Lys-199 residues of albumin with calcium phosphate crystal.

Keywords: Albumin, calcium phosphate, crystallization, nucleation, urolithiasis.

UROLITHIASIS or renal lithiasis is a commonly occurring disease and archaeological evidences stand proof of the fact that mankind has been suffering from kidney and bladder stones for centuries ${ }^{1}$. The five major types of kidney stones found include calcium oxalate, calcium phosphate, uric acid, struvite and cysteine stones. Calcium oxalate $(\mathrm{CaOx})$ is the most common form of kidney stone formation followed by calcium phosphate $(\mathrm{CaP})$, as urine contains ample amounts of calcium which combines with oxalic acid and phosphoric acid to form $\mathrm{CaOx}$ and CaP crystals respectively ${ }^{2,3}$.

Calculi are formed in the urinary tract, including the tubular region of the kidney (nephrolithiasis), ureter (ureterolithiasis) or bladder (cystolithiasis) ${ }^{4}$. This results from several physio-chemical events, including saturation, supersaturation, nucleation, growth, aggregation and retention of the stone-forming constituents ${ }^{5}$. In the supersaturated condition, ions that have been filtered out in the urine by the kidney spontaneously join together to form a solid crystal nucleus through a process called nucleation ${ }^{6}$. In the growth phase, there is further accumulation of ions on the crystal nuclei, thereby resulting in an increase in its size. Aggregation refers to the phase in which the crystals come together to form large aggregates, whereas demineralization is considered a more benign stage of removal of ions from the outer surface of the crystal and hence is considerably important in the prevention of stone formation ${ }^{6}$.

The crystals formed in the supersaturated condition are in constant contact with urine and its various components which have been shown to have an effect on the crystallization process ${ }^{4}$. Inorganic constituents of urine like citrate, magnesium, pyrophosphate and chondroitin sulphate have been known for their inhibitory effects on crystal formation, whereas macromolecules like nephrocalcin, Tamm-Horsfall protein and osteopontin, to name a few, have been identified in the stone matrix and are considered modulators of the crystallization process ${ }^{7-9}$.

Human serum albumin (HSA) is one of the prominent proteins in the renal calculi and its presence has been detected in all types of kidney stone formations ${ }^{7,10-12}$. It is one of the most abundant proteins in the human blood serum as well as urine. It is a transporter of hormones, fatty acids and other compounds, its other well-known functions include maintenance of $\mathrm{pH}$ and oncotic pressure $^{13-15}$. The role of albumin in urolithiasis has been previously studied for its effects in $\mathrm{CaOx}$ crystallization. It preferentially leads to the formation of calcium oxalate dihydrate (COD) crystals over calcium oxalate monohydrate (COM) crystals; these COD crystals are smaller and thus easily removed from the system. Its role as a strong inhibitor of $\mathrm{CaOx}$ crystal aggregation has also been reported $^{16,17}$.

Research on the role of HSA in CaP stone formation is lacking and there is need of more studies in this field as the incidence rate of $\mathrm{CaP}$ stones has increased in the past two decades. The CaP crystals, especially hydroxyapatite, are responsible for nidus formation in the more commonly occurring calcium oxalate kidney stones. Owing to the presence of albumin in the serum, urine and renal calculi, in the present study we have examined the role of albumin in different stages of $\mathrm{CaP}$ crystallization and its association with the crystals through a homogenous system of crystallization and in silico study.

HSA and protein molecular weight marker of medium range for SDS-PAGE were purchased from SigmaAldrich, Bengaluru and MERCK, Bengaluru respectively. All the chemicals used were of analytical grade.

The CaP crystals were mineralized in a homogenous assay system ${ }^{18}$ comprising $50 \mathrm{mM} \mathrm{CaCl} 2 \cdot 2 \mathrm{H}_{2} \mathrm{O}, 50 \mathrm{mM}$ $\mathrm{KH}_{2} \mathrm{PO}_{4}$, Tris buffer $(0.1 \mathrm{MTris}$ base and $210 \mathrm{mM} \mathrm{NaCl}$, $\mathrm{pH}$ 7.4) and distilled water ${ }^{18}$. Varying concentrations of $\operatorname{HSA}(0.5,2,3,4$ and $5 \mathrm{ug} / \mathrm{ml})$ were incorporated in this system to observe the effect on the initial nucleation of $\mathrm{CaP}$ crystals. Each sample was incubated at $37^{\circ} \mathrm{C}$ and centrifuged at $4000 \mathrm{rpm}$ to obtain the pellets.

The preformed $\mathrm{CaP}$ crystals of the nucleated phase were re-suspended in the entire $5 \mathrm{ml}$ assay system with varying concentrations of HSA. Samples were incubated at $37^{\circ} \mathrm{C}$ and centrifuged. Pellets were collected.

For demineralization, various concentrations of HSA with this buffer were added in the preformed $\mathrm{CaP}$ crystals of the initial mineral phase. Each sample was incubated at $37^{\circ} \mathrm{C}$ and centrifuged. Pellets were collected. For the free phase of calcium phosphate mineralization, centrifugation was done at $4000 \mathrm{rpm}$, and the pellet obtained was maintained for further use.

The CaP precipitates of the three phases, viz. nucleation, growth and demineralization were suspended in $0.1 \mathrm{~N} \mathrm{HCl}$, and calcium and phosphate ions present were estimated by alizarin ${ }^{19}$ and Gomori ${ }^{20}$ methods respectively. The percentage inhibition and stimulation of calcium and phosphate was calculated as follows

$$
\% \text { Inhibition/stimulation }=[C-T / C]^{*} 100,
$$

where $T$ is the concentration of calcium or phosphate of the test sample and $C$ is the concentration of calcium or phosphate of the control. 

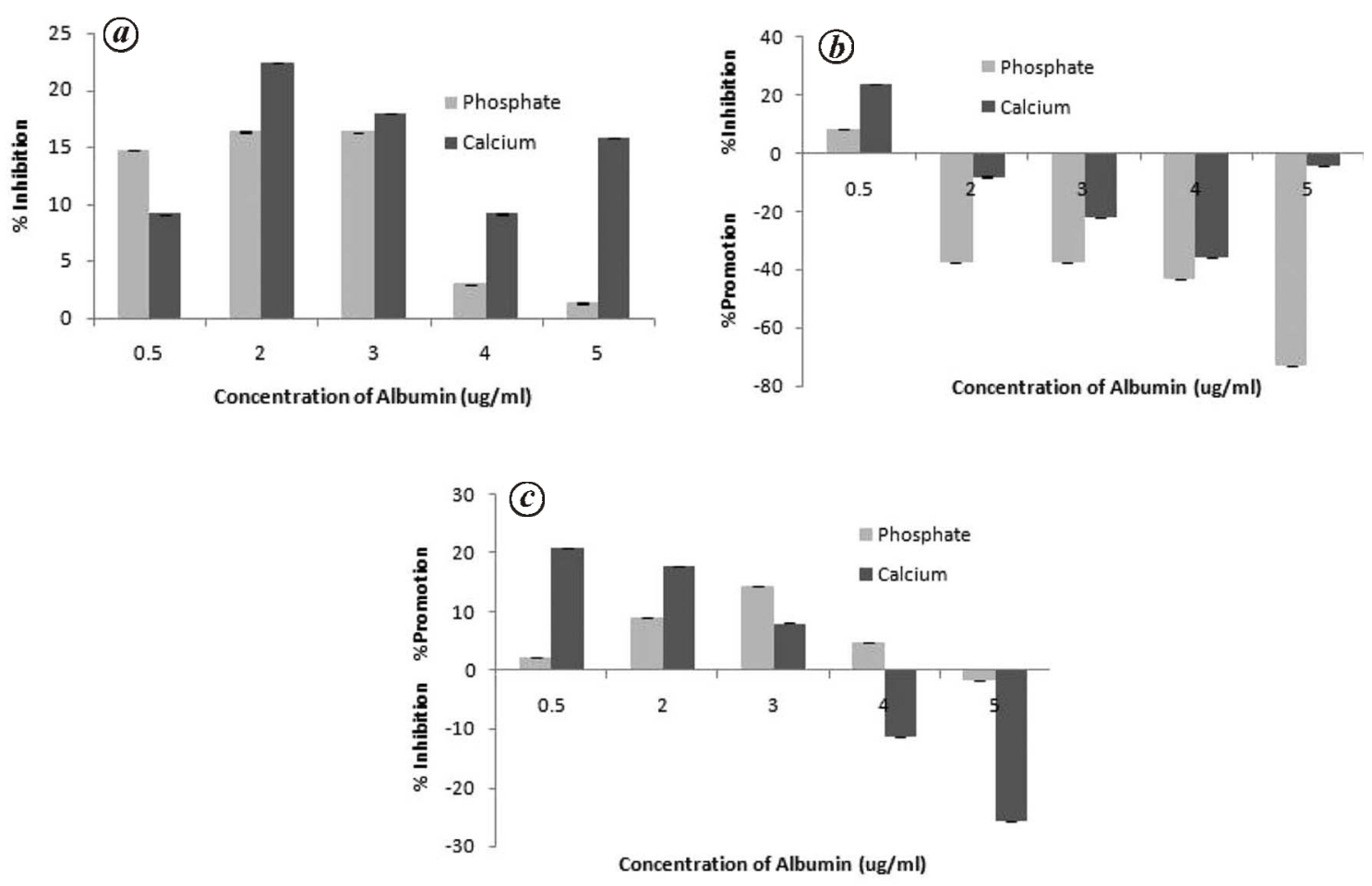

Figure 1. Effect of various concentrations of albumin on (a) nucleation, $(\boldsymbol{b})$ growth and $(\boldsymbol{c})$ demineralization phases of calcium phosphate mineralization.

To study the association of protein with crystals, extraction of protein from $\mathrm{CaP}$ crystals was done using the method of Williams Jr et al. ${ }^{21}$. Briefly, the CaP crystals obtained after nucleation, growth and demineralization of $\mathrm{CaP}$ mineralization in the presence of $5 \mu \mathrm{g} / \mathrm{ml}$ HSA were washed with distilled water. Equal weights of crystals were added with SDS reducing buffer, heated at $95^{\circ} \mathrm{C}$ for $30 \mathrm{~min}$ and centrifuged. The supernatant thus obtained was collected and stored at $-20^{\circ} \mathrm{C}$.

The extracted protein was estimated using the Lowry method $^{22}$. SDS-PAGE ( $10 \%$ resolving gel) was used with loading buffer containing beta-mercaptoethanol. Protein bands were visualized by silver staining.

In order to evaluate the affinity of CaP for HSA, computational docking was performed using Schrödinger GLIDE software LLC: Small-Molecule Drug Discovery Suite 2016-2: Glide, version 7.1 (Schrodinger, LLC, New York, USA). Crystal structure of HSA protein cocrystallized with heteroatoms was downloaded from the Protein Data Bank (PDB ID 1E7H) and ligand (CaP) file was downloaded from NCBI (PubChem ID 24441).

Before docking, the protein structure was prepared by adding hydrogen, removing water molecules and using ligprep function of Glide to generate 32 tautomers at biological $\mathrm{pH}$. CaP ligand was then docked to the HSA protein using extra precision mode (XP-mode) of Glide ${ }^{23}$. Docking scores were quantified in terms of Glide gscore and Glide energy. GlideScore is an empirical scoring function based on Chemscore to compute free binding energies of a ligand. It calculates molecular interacting force fields such as electrostatic, van der Waals, etc. ${ }^{24}$. Glide energy is a specially constructed Coulomb-van der Waals interaction energy score to avoid overly rewarding charge-charge interactions, and is also an important component to derive GlideScore scoring function ${ }^{25}$. Glide Emodel offers many different poses of the docked ligand. In flexible docking, the Glide Emodel score is a combination of energy-grid score, GlideScore and internal strain energy for the corresponding model ${ }^{24}$. The HSA protein has seven binding sites for the binding of ligands; however, the affinity of a particular ligand depends on how well it binds to one or two sites of the protein.

Structure-based, site-directed mutagenesis was done using PyMol to verify the significance of amino acids present in the binding sites of HSA that were involved in ligand binding. The GlideScore obtained after docking simulation was screened to select binding sites for which post-dock analysis was performed. Amino acids with the same volume but opposite physiochemical properties were selected for point mutation ${ }^{26-28}$. Using the protein preparation wizard of glide, protein structure was minimized before docking as it optimizes the bond lengths and angles as well as torsional angles, and rescores the poses using the scaled Coulomb-van der Waals term and GlideScore. Ligplot was used to determine residues involved in hydrogen bonding and hydrophobic interaction 
between protein-ligand complexes within $5 \AA$ radius of the ligand ${ }^{29,30}$.

To further clarify the stereochemistry between the docked $\mathrm{CaP}$ molecule and HSA, we have analysed the post-docked pose of the CaP molecule using UCSFChimera, a program for analysis and visualization of molecular structure.

Albumin exhibited an inhibitory effect on the nucleation of $\mathrm{CaP}$ crystals with maximum inhibition of more than $20 \%$ at $2 \mu \mathrm{g} / \mathrm{ml}$ concentration. Percentage inhibition lowered below $5 \%$ at the maximum concentration of albumin used ( $5 \mu \mathrm{g} / \mathrm{ml})$ (Figure $1 a$ ).

The effect of albumin on the growth of pre-nucleated crystals showed inhibition as well as promotion. Interestingly, lower concentration of albumin $(0.5 \mu \mathrm{g} / \mathrm{ml})$ inhibited the growth of calcium ions to more than $20 \%$, while higher concentration stimulated the growth phase of both ions. The percentage promotion increased with increase in the concentration of albumin. Approximately $73 \%$ promotion was exhibited by albumin at its highest concentration $(5 \mu \mathrm{g} / \mathrm{ml})$ (Figure $1 b)$.

Albumin showed consistent decline in the promotion of $\mathrm{CaP}$ demineralization at its lower concentrations, with maximum percentage promotion of approximately 20 at $0.5 \mu \mathrm{g} / \mathrm{ml}$. With increase in the concentration of albumin, it inhibited the demineralization of calcium and phosphate ions from the preformed crystal nuclei, mounting up to $25 \%$ at $5 \mu \mathrm{g} / \mathrm{ml}$ (Figure $1 c$ ).

Protein content in the CaP crystals for the nucleation phase was the highest at $6.428 \mu \mathrm{g} / \mu \mathrm{l}$ and that for the demineralization phase was $4.856 \mu \mathrm{g} / \mu \mathrm{l}$. The CaP crystals harvested for the growth phase had the lowest protein content of $0.856 \mu \mathrm{g} / \mu \mathrm{l}$.

Protein bands corresponding to $66 \mathrm{kDA}$ were observed in the SDS-PAGE analysis. Evident bands appeared in the lanes for protein extracts of the harvested $\mathrm{CaP}$ crystals obtained at all the three phases studied - nucleation, growth and demineralization. They corresponded to the $66 \mathrm{kDA}$ band for pure albumin extract (Figure 2).

Docking was done to produce many conformations of the protein-ligand complex, among which at least one native-like conformation was found. Lowest binding energy conformation is considered the most suitable docking pose; therefore, active sites 1 and 7 were selected for further studies. Moreover, Glide gscore, Glide energy and Glide emodel were high for binding sites 1 and 7 (Table 1). In the post-dock analysis we found that the ligands for active sites 1 and 7 formed hydrogen bonds with Tyr, Lys and Arg residues. However, other sites too showed hydrogen bonding with the ligands, but their cumulative docking scores were low and hence were not considered for the study. As the ligand size was small, docking score of all HSA sites was low ranging between -0.5 and -4 .

Post-dock analysis by point mutation was done for active sites 1 and 7 . In site 1 the ligand binds with Tyr161, while in site 7 it binds with Arg-222 and Lys-195 and Lys-199 (Figure 3). Both Lys and Arg are basic and hydrophobic in nature; therefore, they were mutated with Glu and Ile respectively. Tyr is a neutral amino acid and thus we replaced it with Arg (hydrophilic and basic), Asp (hydrophilic and acidic) and Phe (hydrophobic and aliphatic).

After mutation, a significant increase in GlideScore and energy was observed in the case of Tyr-161 at site 1 . At active site 7 mutating Arg-222 with Glu, there was a minor change in the GlideScore $(-3.935$ to $-3.651 \mathrm{kcal} / \mathrm{mol})$ and glide energy ( -20.068 to $-13.487 \mathrm{kcal} / \mathrm{mol}$ ) (Table 2). Ligplot was used to further verify the docking result for sites 1 and 7 . At site $1 \mathrm{CaP}$ formed a hydrogen bond with Tyr-161 and hydrophobic bonds with Tyr-138, Lys137, Met-123 and Phe-134 (Figure 4). While at site 7 it formed hydrogen bonds with Lys-195, Lys-199 and Arg222, and hydrophobic bonds with Trp-214. LigPlot result of mutation of Arg-222 to Ile for site 7 did not show any interaction with $\mathrm{CaP}$. Interestingly, $\mathrm{CaP}$ was found to bind with Arg-117 by hydrogen bond, while hydrophobic contact was observed with Met-123, Leu-182 and Tyr161 (Figure 5).

Urolithiasis, the formation of stones in the urinary tract, urethra and kidneys, is a prevalent disease worldwide. It is largely influenced by demography and is seen to be more frequent in men ${ }^{31,32}$. Stone matrix comprising $90 \%$ inorganic matter and 2-5\% organic content reveals albumin to be one of its constituent proteins in almost all urinary stones, regardless of the crystal type ${ }^{33}$.

Table 1. Results of docking study for seven binding sites of HSA protein with calcium phosphate structure screened by three score functions for analysis

\begin{tabular}{lccc}
\hline Active site & $\begin{array}{c}\text { GlideScore } \\
(\mathrm{kcal} / \mathrm{mol})\end{array}$ & $\begin{array}{c}\text { Glide energy } \\
(\mathrm{kcal} / \mathrm{mol})\end{array}$ & $\begin{array}{c}\text { Glide Emodel } \\
(\mathrm{kcal} / \mathrm{mol})\end{array}$ \\
\hline Active site 1 & -3.268151 & -19.683523 & -21.376899 \\
Active site 2 & -1.302795 & -8.681075 & -10.302306 \\
Active site 3 & -1.659011 & -9.655592 & -9.926723 \\
Active site 4 & -1.304081 & -5.288570 & -5.962102 \\
Active site 5 & -0.663181 & -3.834067 & 0.000000 \\
Active site 6 & -3.016308 & -10.071202 & -11.403970 \\
Active site 7 & -3.935360 & -20.068924 & -20.717667 \\
\hline
\end{tabular}

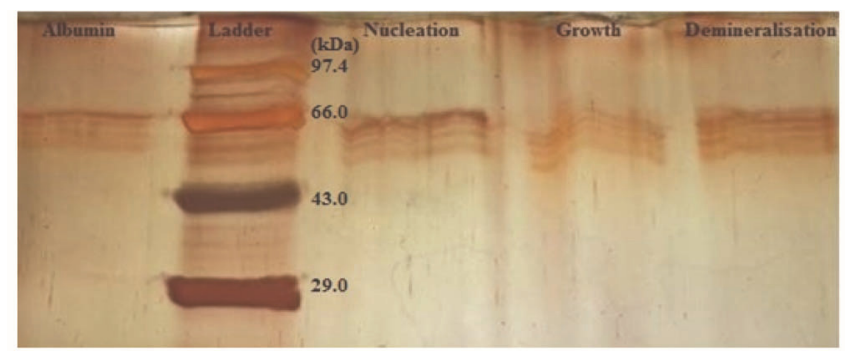

Figure 2. SDS-PAGE analysis showing the presence of HSA protein in all the lanes - nucleation, growth and demineralization. 

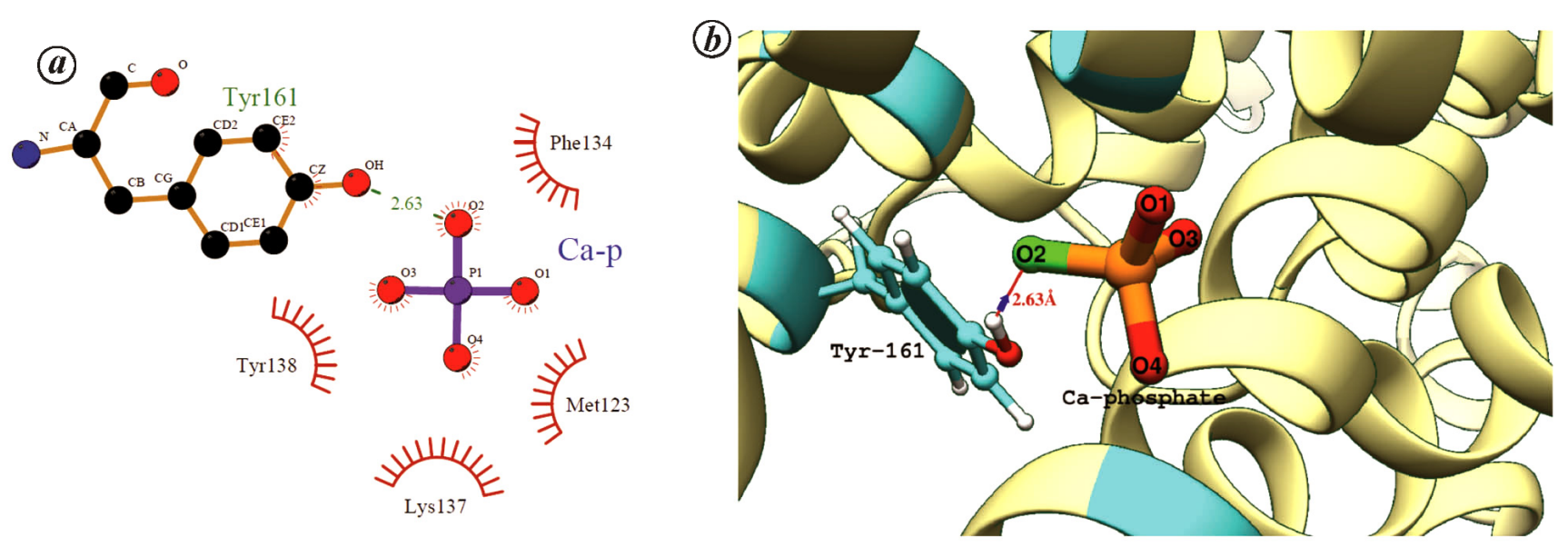

Figure 3. a, Protein-ligand interaction analysed by Ligplot. Calcium phosphate (ligand) binding in pocket 1 with Tyr-161 via hydrogen bond while Phe-134, Met-123, Lys-137 and Tyr-138 show hydrophobic contacts. $\boldsymbol{b}$, Protein-ligand interaction analysed by Chimera. Calcium phosphate (ligand) binding in pocket 1 with Tyr-161 via hydrogen bond (labelled red). The oxygen atom forming hydrogen bond with HSA amino acid Tyr161 is coloured green, while the non-hydrogen bonding oxygen atoms are coloured red. The directionality of hydrogen bond is represented by blue arrow, with arrow base representing donor and head representing acceptor of the hydrogen bond.
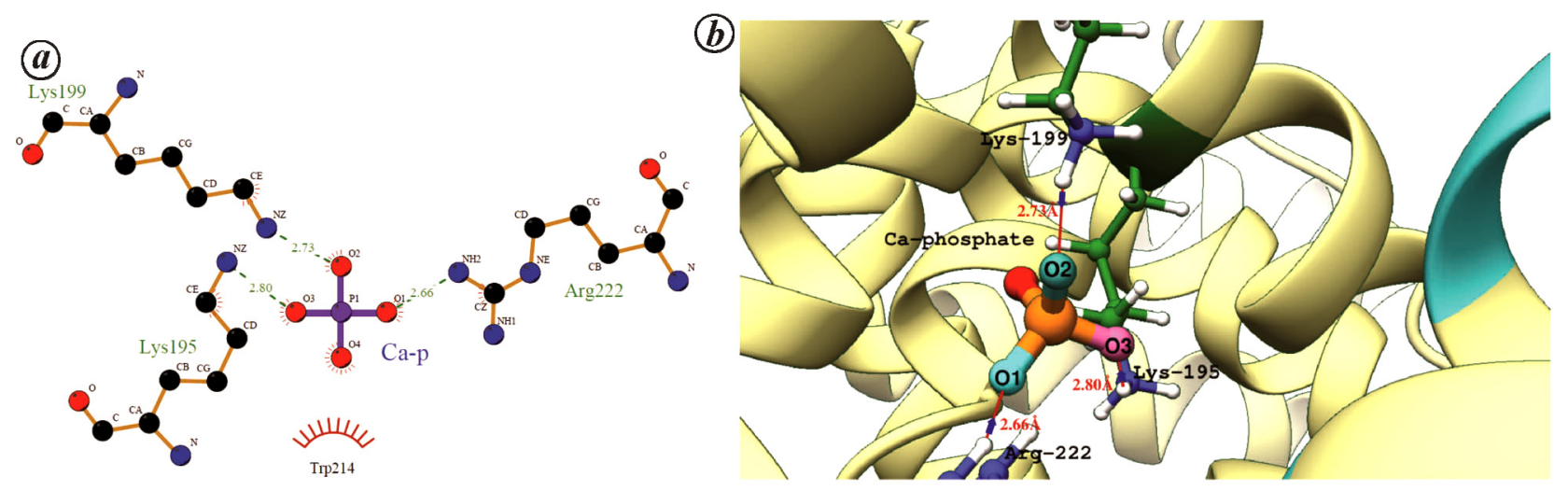

Figure 4. $\boldsymbol{a}$, Calcium phosphate (ligand) binding in pocket 7 with Lys-195, Lys-199 and Arg-222 via hydrogen bond, while Trp-214 shows hydrophobic contacts. b, Calcium phosphate (ligand) binding in pocket 7 with Lys-195, Lys-199 and Arg-222 via hydrogen bond (labelled red). The oxygen atoms forming hydrogen bonds with HSA amino acids Lys-195, Lys-199 and Arg-222 are coloured pink, sea blue and magenta respectively, while the non-hydrogen bonding oxygen atom is coloured red. The directionality of hydrogen bonds are represented by blue arrows, with arrow base representing donor and head representing acceptor of the hydrogen bonds.
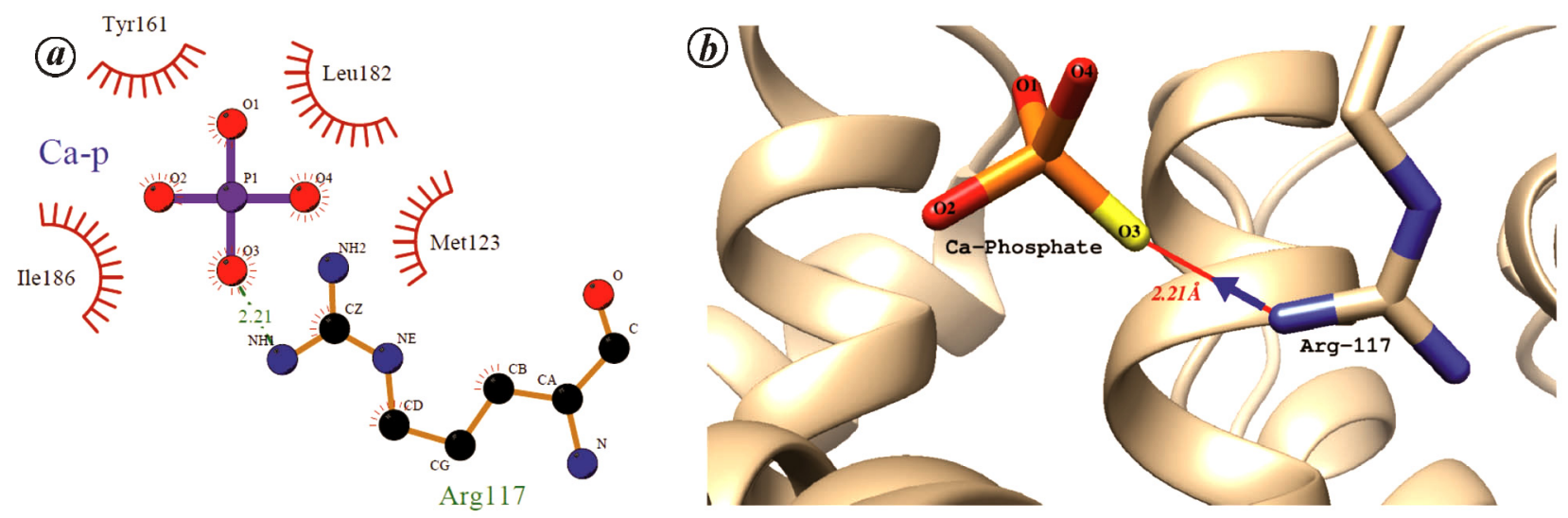

Figure 5. $\boldsymbol{a}$, Mutation of Arg-186 to Ile show binding of human serum albumin to calcium phosphate (ligand) binding in pocket 7 with Arg-117 via hydrogen bond, while Met-123, Ile-186, Tyr-161 and Leu-182 show hydrophobic contacts. $\boldsymbol{b}$, Mutation of Arg-186 to Ile showing binding of human serum albumin to calcium phosphate (ligand) binding in pocket 7 with Arg-117 via hydrogen bond (labelled red). The oxygen atom forming hydrogen bond with HSA amino acid Arg-117 is coloured yellow, while the non-hydrogen bonding oxygen atoms are coloured red. The directionality of hydrogen bond is represented by blue arrow, with arrow base representing donor and head representing acceptor of the hydrogen bond. 
RESEARCH COMMUNICATIONS

Table 2. Docking results of HSA protein after site-directed mutagenesis for binding sites 1 and 7

\begin{tabular}{lllll}
\hline Active site of HSA & \multicolumn{1}{c}{ Point mutation } & GlideScore $(\mathrm{kcal} / \mathrm{mol})$ & Glide energy (kcal/mol) & Glide Emodel (kcal/mol) \\
\hline \multirow{2}{*}{ Active site 1 } & mut_Tyr-161_to_Arg & -3.341 & -18.546 & -20.608 \\
& mut_Tyr-161_to_Asp & -4.01 & -21.93 & -21.568 \\
Active site 7 & mut_Tyr-161_to_Phe & -4.046 & -13.487 & -22.488 \\
& mut_Arg-222_to_Glu & -3.651 & -14.947 & -14.526 \\
& mut_Arg-222_to_Ile & -3.653 & -13.723 & -15.933 \\
& mut_Lys-195_to_Glu & -3.053 & -17.87 & -14.04 \\
& mut_Lys-195_to_Ile & -3.882 & -15.724 & -13.712 \\
& mut_Lys-199_to_Glu & -3.109 & -17.478 \\
\hline
\end{tabular}

In the present study, the effect of albumin on the three phases of stone development, i.e. nucleation, growth and demineralization was observed. Protein extraction from crystals obtained from the three phases of $\mathrm{CaP}$ mineralization and their estimation suggested the presence of albumin in crystal precipitates. Protein bands of $66 \mathrm{kDa}$ were observed through SDS-PAGE analysis. Albumin showed modulation of calcium and phosphate ions in all the three stages of its mineralization. It had an overall inhibitory effect in nucleation at all concentrations used, i.e. from 0.5 to $5 \mu \mathrm{g} / \mathrm{ml}$. In growth phase, albumin showed dual nature, i.e. low concentrations showed inhibition, while at higher concentrations it promoted crystal growth. Albumin was also seen to display a dual nature in the demineralization of CaP crystals. At low concentration, it promoted the demineralization of calcium and phosphate ions from the crystals, while it inhibited demineralization of calcium ions from the crystal nuclei at $4 \mathrm{ug} / \mathrm{ml}$ and phosphate ions at its $5 \mu \mathrm{g} / \mathrm{ml}$ concentration.

The findings of the present study and those previously done support Albumin's role in stone formation, though further investigations to elucidate the exact role of the protein are required. The present study reveals only inhibitory role of albumin in nucleation while putative dual nature was observed in the growth and demineralization phases.

HSA possesses seven different ligand-binding sites with different affinities for different ligands. Docking experiments revealed that two of these binding sites (sites 1 and 7) exhibit relatively higher affinity for $\mathrm{CaP}$ compared to other binding sites. Post-docking analysis of ligand-protein interactions at these binding sites, confirmed the formation of hydrogen bonds between active site residues and ligands. Since docking results indicated higher affinities of binding sites 1 and 7, we further structurally mutated hydrogen bond-forming residues in these binding sites by replacing the corresponding amino acid residue with another amino acid. For all these point substitutions, volume of the corresponding amino acid was considered and replacements were done with amino acids that occupy similar volume in the void during replacement. Replacement of Arg was done with Ile and Glu. As
Arg is a hydrophilic and basic amino acid, it was replaced by acidic amino acid Glu and hydrophobic and aliphatic amino acid Ile. Similarly Lys, a hydrophilic and basic amino acid was replaced with Glu and Ile. Tyr, a neutral amino acid, was replaced by Arg (hydrophilic and basic), Asp (hydrophilic and acidic) and Phe (hydrophobic).

After performing structural site-directed mutagenesis at specific amino acid positions, the mutated structures were prepared and minimized with GLIDE protein preparation and minimization tool. Then each mutated structure was docked with $\mathrm{CaP}$ crystals. Post-mutational docking of $\mathrm{CaP}$ crystal with HSA computed with significant decrease in Glide docking score and Emodel values at binding site 7. The GlideScore and Emodel values were -3.053, -14.04 and $-2.785,-17.478 \mathrm{kcal} / \mathrm{mol}$ for mutations at Lys-195 and Lys-199 respectively, indicating that the ligand probably binds to binding site 7 of HSA. Postdocking studies of these mutated structures also demonstrated a decrease in the formation of hydrogen bonds in them.

Albumin showed modulatory effects in all the phases of renal calculi formation. The inhibitory effects observed in the nucleation phase are suggestive of a possible role in preventing formation of renal calculi, while stimulatory effect at higher concentrations in the growth and demineralization phases indicate a positive contribution towards kidney stone development. Hence, as previously reported, albumin showed modulatory effects in $\mathrm{CaP}$ crystallization. Moreover, its modulation of $\mathrm{CaOx}$ crystallization has also been reported ${ }^{16,17}$. The findings of the present study reveals the possible role of albumin in stone formation, though further studies are required. This study reveals inhibitory role of albumin in nucleation and a putative dual nature in the growth and demineralization phases of calcium phosphate crystallization.

Conflict of interest: The authors declare that they have no conflict of interest.

1. López, M. and Hoppe, B., History, epidemiology and regional diversities of urolithiasis. Pediatr. Nephrol., 2010, 25(1), 49-59. 


\section{RESEARCH COMMUNICATIONS}

2. Coe, F. L., Parks, J. H. and Asplin, J. R., The pathogenesis and treatment of kidney stones. N. Engl. J. Med., 1992, 327(16), 11411152 .

3. Yuvarani, T., Manjula, K. and Gopu, P., Growth characterization of calcium, hydrogen phosphate dihydrate crystals influenced by Costus igneus aqueous extract. Int. J. Pharm. Pharm. Sci., 2017, 9(5), 173-178.

4. Aduayi, O. S. and Famurewa, O. C., Cystolithiasis with coexisting nephrolithiasis: a radiodiagnostic discovery in an adult Nigerian male with lower urinary tract symptoms. J. Med. Invest. Pract., 2015, 10, 30-32.

5. Siener, R. and Hesse, A., The effect of a vegetarian and different omnivorous diet on urinary risk factors for uric acid stone formation. Eur. J. Nutr., 2003, 42(6), 332-337.

6. Evan, A. P. et al., Mechanism of formation of human calcium oxalate renal stones on Randall's plaque. Anat. Rec., 2007, 290, 1315-1323.

7. Kaneko, K., Kobayashi, R., Yasuda, M., Izumi, Y., Yamanobe, T and Shimizu, T., Comparison of matrix proteins in different types of urinary stone by proteomic analysis using liquid chromatography-tandem mass spectrometry. Int. J. Urol., 2012, 19, 765-772.

8. Jaggi, M., Nakagawa, Y., Zipperle, L. and Hess, B., TammHorsfall protein in recurrent calcium kidney stone formers with positive family history: abnormalities in urinary excretion, molecular structure and function. Urol. Res., 2007, 35(2), 55-62.

9. Devuyst, O., Dahan, K. and Prison, Y., Tamm-Horsfall protein or uromodulin: new ideas about an old molecule. Nephrol. Dial. Transplant, 2015, 20, 1290-1294.

10. Terkeltaub, R., Santoro, D., Mandel, G. and Mandel, N., Serum and plasma inhibit neutrophil stimulation by hydroxyapatite crystals, evidence that serum $\alpha_{2}$-HS glycoprotein is a potent and specific crystal-bound inhibitor. Arthritis Rheum., 1988, 31, 10811089.

11. Atmani, F. and Khan, S., Modulators of urinary stone formation. Urol. Int., 2002, 68, 54-59.

12. Dussol, B. et al., Analysis of the soluble organic matrix of five morphologically different kidney stones. Urol. Res., 1995, 23, 4551.

13. Quinlan, G. J., Martin, G. S. and Evans, T. W., Albumin: biochemical properties and therapeutic potential. Hepatology, 2005, 41, 1211-1219.

14. Hankins, J., The role of albumin in fluid and electrolyte balance. J. Infus. Nurs., 2006, 29, 261-265.

15. Sitar, M. E., Aydin, S. and Cakatay, U., Human serum albumin and its relation with oxidative stress. Clin. Lab., 2013, 59, 945952

16. Cerini, C. et al., Nucleation of calcium oxalate crystals by albumin: involvement in the prevention of stone formation. Kidney Int., 1999, 55, 1776-1786.

17. Liu, J., Jiang, H. and Liu, X., How does bovine serum albumin prevent the formation of kidney stone? A kinetics study. J. Phys. Chem. B., 2006, 110, 9085-9089.

18. Kabra, S. G., Kabra, V., Banerji, P., Jain, L. K., Bhargava, A. and Chaturvedi, R. P., Inhibitors of in vitro mineralization from flexor tendons of rabbits and their role in biological mineralization. Indian J. Exp. Biol., 1978, 16, 212-217.

19. Natelson, S. and Penniall, R., Colorimetric estimation of ultramicro quantities of calcium in human serum as complex with alizarin. Anal. Chem., 1955, 27, 434-437.

20. Gomori, G., A modification of the colorimetric phosphorus determination for use with the photoelectric colorimeter. J. Lab. Clin. Med., 1942, 27, 955-960.

21. Williams, J. C. Jr, Zarse, C. A., Jackson, M. E., Witzmann, F. A and McAteer, J. A., Variability of protein content in calcium oxalate monohydrate stones. J. Endourol., 2006, 20(8), 560564
22. Lowry, O. H., Rosbrough, N. J., Farr, A. L. and Randall, R. J., Protein measurement with the Folin Phenol reagent. J. Biol. Chem., 1951, 193, 265-275.

23. Bamane, R., Chitre, T. and Rakholiya, V. K., Molecular docking studies of quinoline-3-carbohydrazide as novel PTP1B inhibitors as potential antihyperglycemic agents. Der Pharma Chem., 2011, 3, 227-237.

24. Friesner, R. A., Banks, J. L., Murphy, R. B., Halgren, T. A. and Klicic Glide, J. J., A new approach for rapid, accurate docking and scoring. 1. Method and assessment of docking accuracy. J. Med. Chem., 2004, 47, 1739-1749.

25. Balaji, B. and Ramanathan, M., Prediction of estrogen receptor $\beta$ ligands potency and selectivity by docking and MM-GBSA scoring methods using three different scaffolds. J. Enzyme Inhib. Med. Chem., 2012, 27, 832-844.

26. Brennan, S. and Carrell, S., $\alpha_{1}$-Antitrypsin Christchurch, 363 $\mathrm{Glu} \rightarrow$ Lys: mutation at the $\mathrm{P}^{\prime} 5$ position does not affect inhibitory activity. Biochim. Biophys. Acta, 1986, 873, 13-19.

27. Nishizawa, K., Nishizawa, M., Gnanasambandam, R., Sachs, F., Sukharev, S. I. and Suchyna, T. M., Effects of Lys to Glu mutations in GsMTx4 on membrane binding, peptide orientation, and self-association propensity, as analyzed by molecular dynamics simulations. Biochim. Biophys. Acta, 2015, 1848, $2767-$ 2778.

28. Shinbrot, E. et al., Exonuclease mutations in DNA polymerase epsilon reveal replication strand specific mutation patterns and human origins of replication. Genome Res., 2014, 24, 1740 1750.

29. Cherepanov, D. et al., Reduction and protonation of the secondary quinone acceptor of Rhodobactersphaeroides photosynthetic reaction center: kinetic model based on a comparison of wild-type chromatophores with mutants carrying Arg $\rightarrow$ Ile substitution at sites 207 and 217 in the L-subunit. Biochim. Biophys. Acta, 2000, 1459, 10-34.

30. Ishtikhar, M., Ali, M. S., Atta, M., Nigam, L., Subbarao, N. and Khan, R. H., Temperature dependent rapid annealing effect induces amorphous aggregation of human serum albumin. $J$. Lumin., 2015, 167, 399-407.

31. Stamatelou, K. K., Francis, M. E., Jones, C. A., Nyberg, L. M. and Curhan, G. C., Time trends in reported prevalence of kidney stones in the United States: 1976-1994. Kidney Int., 2003, 63, 1817-1823.

32. Sourcie, J. M., Thun, M. J., Coates, R. J., McClellan, W. and Austin, H., Demographic and geographic variability of kidney stones in the United States. Kidney Int., 1994, 46, 893-899.

33. Alelign, T. and Petros, B., Kidney stone disease: an update on current concepts. Adv. Urol., 2018, 2018, 3068365.

Received 1 August 2018; revised accepted 3 July 2019

doi: $10.18520 / \mathrm{cs} / \mathrm{v} 117 / \mathrm{i} 6 / 1083-1089$ 